RESPIRATORY INFECTION

\title{
Recovery of the ciliated epithelium following acute bronchiolitis in infancy
}

\author{
J Y W Wong, A Rutman, C O'Callaghan
}

Thorax 2005;60:582-587. doi: 10.1136/thx.2004.024638

See end of article for authors' affiliations

Correspondence to:

Professor C O'Callaghan Department of Child Health, University of Leicester, School of Medicine, Robert Kilpatrick Clinical Sciences Building, Leicester Royal Infirmary, $\mathrm{P} O \mathrm{Box} 65$, Leicester LE2 7LX, UK; ajb64@le.ac.uk

Received 10 March 2004 Accepted 20 April 2005
Background: Little is known about the longitudinal changes in the ciliated respiratory epithelium of infants following viral bronchiolitis. A study was undertaken to investigate the time required for the ciliated epithelium to return to normal following bronchiolitis in infants treated with inhaled steroids or placebo. Methods: Thirty one previously healthy term infants were studied as part of a clinical trial to determine the effect of 12 weeks of treatment with inhaled fluticasone (FP) or placebo via a spacer device (17 FP, 14 placebo). Nineteen healthy children aged 0-6 years previously studied in our department were used as controls. Nasal biopsy specimens were taken from infants with bronchiolitis and ciliary beat frequency (CBF) was measured before treatment and repeated 3, 6, 12, and 24 weeks later. The epithelial ultrastructure was examined by transmission electron microscopy and a normal errors mixed model based on normal controls was used to examine the time for cilia to return to normal in bronchiolitic infants.

Results: The mean CBF of infants with bronchiolitis (in $\mathrm{Hz}$ ) at weeks $0,3,6,12$, and 24 were $0.5(\mathrm{n}=4)$, $10.9(n=4), 12.0(n=9), 11.9(n=8)$, and $12.1(n=7)$ in the placebo group and $10.6(n=6), 11.4$ $(n=9), 8.8(n=8), 10.9(n=4)$, and $13.2(n=7)$ in the FP group. The time for the epithelial ultrastructure to normalise was as follows: epithelial integrity score (13.1 weeks), \% ciliated cells with loss of cilia (14.0 weeks), and \% epithelial cells with abnormalities in projection (16.7 weeks) or mitochondria (15.9 weeks). Inhaled steroids had no significant effects on CBF or epithelial ultrastructure.

Conclusion: Ciliary loss and epithelial abnormalities persist on average for 13-17 weeks following acute bronchiolitis in infancy.
A $\mathrm{n}$ intact ciliated respiratory epithelium is an important part of the host's defence. The ciliated epithelium of the upper respiratory tract of young children is frequently exposed to viral infections and abnormalities of ciliary function and ultrastructure have been reported in children aged 2-6 years who suffered from a single or recurrent viral upper respiratory tract infections. ${ }^{12}$

Viral upper respiratory tract infections are most often associated with minor and transient respiratory symptoms, but respiratory symptoms may persist for a considerable time in infants following viral bronchiolitis. The commonest virus responsible for bronchiolitis is the respiratory syncytial virus (RSV) which has been shown in vitro to cause ciliary loss during the acute infection. ${ }^{3}$ Despite the high frequency of acute bronchiolitis during infancy and the protracted respiratory problems following infection, little is known of the rate of the subsequent recovery of the respiratory epithelium. Indeed, we are unaware of any study that has investigated the longitudinal recovery of the nasal epithelium following viral bronchiolitis in infancy.

We recently reported the lack of effect of inhaled fluticasone propionate (FP) in reducing clinical symptoms following bronchiolitis in infancy. ${ }^{4}$ As part of this randomised placebo controlled trial, we measured the ciliary beat frequency (CBF) and evaluated the ultrastructure of the nasal respiratory epithelium of infants following discharge and $0,3,6,12$, and 24 weeks later using light and transmission electronic microscopy. In this paper we describe the nature and duration of the damage to the nasal ciliated epithelium seen in these infants in comparison with our published normal values. ${ }^{5}$

\section{METHODS}

\section{Patients}

Forty eight infants admitted to hospital with acute bronchiolitis, ${ }^{4}$ according to criteria suggested by Court, ${ }^{6}$ were recruited immediately before discharge from hospital. Recruitment was part of a double blind, randomised, placebo controlled trial ${ }^{4}$ that was designed to determine whether infants receiving an inhaled steroid (FP) for 3 months had fewer respiratory symptoms following bronchiolitis than those receiving placebo. All infants were admitted to the Leicester Royal Infirmary with their first episode of a lower respiratory tract infection. They were otherwise healthy infants who had not previously required hospital admission and were not on regular medication. In particular, they had no previous history of wheeze or respiratory illnesses apart from transient upper respiratory symptoms. Exclusion criteria were: birth before 36 weeks of gestation; congenital heart disease or syndromic abnormalities; established systemic or chronic illnesses; treatment with corticosteroids or mechanical ventilation before entering the study. Nasopharyngeal aspirates were sent for immunofluorescent study and viral culture. Ethical approval was also obtained to take nasal brush biopsies over the 6 months following their bronchiolitis.

Nasal epithelial biopsies were obtained in 31 infants. Nineteen healthy children previously studied in our department who had no known respiratory or ENT diseases were used as controls. These children were aged 0-6 years (mean 3.4); five were infants (mean age 0.8 years). Normal values on epithelial and ciliary ultrastructure examined by transmission electron microscopy (TEM) and CBF measured by a high speed video system from nasal epithelium of these healthy controls have previously been published. ${ }^{5}$ As the CBF of the infants with bronchiolitis in this study was measured using the modified photodiode technique, ${ }^{5}$ only the TEM data of

Abbreviations: $C B F$, ciliary beat frequency; FP, fluticasone propionate; RSV, respiratory syncytial virus; TEM, transmission electron microscopy 
the control group were used for comparison with the bronchiolitis group.

The study was approved by the Leicestershire ethical committee and written informed consent was obtained.

\section{Study design}

Infants with bronchiolitis were randomised to receive either $150 \mu \mathrm{g}$ FP or placebo twice daily using a Babyhaler spacer and face mask device for 3 months. Nasal ciliary brushings were performed using a small bronchoscopy brush at weeks 0 (recruitment), 3, 6, 12 (end of treatment), and 24. Samples were placed in M199 solution and CBF was measured within 4 hours at $36.5-37^{\circ} \mathrm{C}$ using an interference contrast $\times 100$ oil immersion lens. Ciliated edge images were projected by video camera to a high resolution video screen and CBF was determined using the modified photodiode technique. ${ }^{7} \mathrm{~A}$ minimum of four readings from four different epithelial edges were obtained from each sample and the standard deviation (SD) of CBF was calculated.

The remainder of the sample was fixed in $4 \%$ gluteraldehyde for TEM examination. This was processed through to resin by standard techniques. ${ }^{8}$ Ultrathin sections were cut at $70 \mathrm{~nm}$, collected on 200 mesh thin bar copper grids, stained in $1 \%$ uranyl acetate and counterstained in Reynold's lead phosphate before assessment by TEM in a blind fashion.

The numbers of ciliated cells, mucous cells, and dead cells were expressed as a percentage of all cells examined. Epithelial integrity was assigned a score of 0-5 according to the method of Tsang et al ${ }^{9}$ based on: loss of cilia from ciliated cells from 0 (fully ciliated) to 3 (few cilia visible); projection of cells from the epithelial edge from 0 (normal alignment) to 3 (projected from edge with minimal contact to adjacent epithelial cells); cytoplasmic blebbing $(0=$ absent, $1=$ minor, $2=$ major $)$; mitochondrial damage $(0=$ absent, $1=$ present $)$. Percentages were calculated for the number of cells with either loss of cilia, cellular projections, cytoplasmic blebbing, mitochondrial damage, the cellular appearance of single detached cells (normal, round or denatured), and those with microtubular or dynein arm defects. The degree of abnormality was scored for cilia projections and extent of cilia loss on epithelial cells as follows: 0 (normal), + (slightly abnormal), $++(<50 \%$ abnormality $),+++(>50 \%$ abnormality $)$.

\section{Statistical methods}

Normality of CBF data was tested using the Shapiro-Francia W test. Group mean values (with 95\% confidence intervals) for each treatment group were calculated for each batch of biopsy specimens taken between weeks 0 and 24 . The values at week 0 were compared with those at week 12 (end of treatment) using the paired sample Student's $t$ test with Bonferroni correction. TEM findings were reported as group mean with standard deviations (SD). A regression analysis of the TEM data on healthy infants ${ }^{5}$ was used to estimate the mean level of each variable at 6 months (the mean age at which infants in the bronchiolitis trial would have finished their 3 months of inhaled treatment). The bronchiolitis trial TEM data were log transformed to improve normality. A normal errors mixed model ${ }^{10}$ that allowed for within and between subject variability was used to model the change over time only in the bronchiolitis TEM data. The fit of this model was used to predict the age at which each trial subject reached the predicted 6 month level of the healthy infants. Statistical analyses were performed using Stata (Stata Corporation, Texas, USA).

\section{RESULTS}

Twenty four infants in each treatment group entered the study during the same bronchiolitis season. At the beginning of the trial, parental consent for nasal ciliary biopsy specimens to be taken was obtained from 12 infants in the placebo group and 16 in the FP group. Following this, consent was obtained at a subsequent visit from three additional infants (two on placebo and one on FP). Baseline ciliary specimens were not obtained from these infants. The final cohort of patients consisted of 31 infants (17 FP and 14 placebo).

The demographic data were similar in the two groups and are shown in table 1. During the 12 week trial period one infant in the FP group was withdrawn on day 10 due to noncompliance with inhaler therapy. Biopsies were not obtained on every occasion for various circumstantial reasons (for example, if the infant was asleep). This resulted in biopsy specimens being obtained from 10 in the placebo group and eight in the FP group on all four occasions during the 12 week treatment period. The parents of one infant in the FP group declined a post-treatment biopsy at 24 weeks.

\section{Light microscopy findings}

A total of 104 epithelial edges were examined. Excluding the first biopsy specimen taken when infants were recovering from acute bronchiolitis, five infants on one occasion and one infant on two occasions in the FP group had evidence of an upper respiratory tract infection on the day of the nasal biopsy (three during the treatment period and four during the follow up period). All but one of the samples, taken while a child had clinical evidence of an upper respiratory tract infection, revealed extensive ( $>90 \%$ ) denudation of epithelial surfaces.

\begin{tabular}{|c|c|c|}
\hline & $\begin{array}{l}\text { FP } \\
(n=17)\end{array}$ & $\begin{array}{l}\text { Placebo } \\
(n=14)\end{array}$ \\
\hline $\operatorname{Sex}(M / F)$ & $9 / 8$ & $4 / 10$ \\
\hline Gestation (weeks) & $39.4(38.6 \text { to } 40.1)^{*}$ & $39.8(38.9 \text { to } 40.7)^{*}$ \\
\hline Weight centile & $48.0(32.3 \text { to } 63.7)^{*}$ & $34.7(18.3 \text { to } 51.2)^{*}$ \\
\hline Age (weeks) & $15.6(10.2 \text { to } 21.0)^{*}$ & $15.0(10.2 \text { to } 19.8)^{\star}$ \\
\hline \multicolumn{3}{|l|}{ Race } \\
\hline White & $15(88.2 \%)$ & $14(100 \%)$ \\
\hline Others & $2(11.8 \%)$ & $0(0 \%)$ \\
\hline Eczema on exam & $5(17.6 \%)$ & $5(35.7 \%)$ \\
\hline Asthma in mother & $2(11.8 \%)$ & $4(28.6 \%)$ \\
\hline Passive smoking & $10(58.8 \%)$ & $7(50.0 \%)$ \\
\hline RSV & $8(47.1 \%)$ & $6(42.9 \%)$ \\
\hline Other virus (CMV) & $1(5.9 \%)$ & $0(0 \%)$ \\
\hline Days in hospital & $3.4(2.4 \text { to } 4.3)^{*}$ & $3.6(2.4 \text { to } 4.8)^{*}$ \\
\hline
\end{tabular}


Table 2 Ciliary beat frequency (CBF) and standard deviation (CBF SD) in $\mathrm{Hz}$

\begin{tabular}{|c|c|c|c|c|c|}
\hline \multirow[b]{2}{*}{ Abnormalities } & \multicolumn{5}{|c|}{ Weeks from baseline } \\
\hline & 0 & 3 & 6 & 12 & 24 \\
\hline \multicolumn{6}{|l|}{$\mathrm{CBF}$} \\
\hline Placebo & $10.5(3.2$ to 17.9$)$ & 10.9 (8.8 to 13.0$)$ & 12.0 (10.4 to 13.7$)$ & 11.9 (10.6 to 13.2 ) & 12.1 (10.3 to 13.8$)$ \\
\hline $\mathrm{FP}$ & 10.6 (7.0 to 14.3$)$ & $11.4(9.9$ to 13.0$)$ & $8.8(5.5$ to 12.1$)$ & 10.9 (8.8 to 13.1$)$ & 13.2 (11.8 to 14.6$)$ \\
\hline Total & 10.6 (5.3 to 14.8$)$ & $11.3(9.0$ to 13.5$)$ & 10.5 (8.0 to 13.5$)$ & 11.6 (9.7 to 13.2$)$ & $12.6(10.4$ to 13.9$)$ \\
\hline \multicolumn{6}{|l|}{ CBF SD } \\
\hline Placebo & $1.6(-0.1$ to 3.3$)$ & $2.7(0.5$ to 4.9$)$ & $1.8(1.0$ to 2.6$)$ & 1.9 (1.4 to 2.3$)$ & $1.6(1.1$ to 2.0$)$ \\
\hline $\mathrm{FP}$ & $1.6(1.1$ to 2.2$)$ & 2.0 (1.5 to 2.5$)$ & 1.8 (1.0 to 2.5$)$ & $2.0(1.0$ to 2.9$)$ & $1.3(1.1$ to 1.6$)$ \\
\hline Total & $1.6(0.9$ to 2.2$)$ & $2.2(1.2$ to 3.3$)$ & $1.8(0.0$ to 3.1$)$ & $1.9(1.3$ to 2.5$)$ & $1.5(1.1$ to 1.9$)$ \\
\hline
\end{tabular}

\section{Ciliary beat frequency (CBF)}

A significant number of biopsy samples, particularly at an early stage following bronchiolitis, had denuded epithelial surfaces making CBF measurements impossible. The proportions of biopsy samples where CBF could be measured taken at $0,3,6,12$, and 24 weeks were $4 / 12,4 / 13,9 / 13,8 / 13$, and $7 /$ 12 in the placebo group and 6/16,9/15, 8/14, 4/11, and 7/13 in the FP group. As there were no obvious differences in $\mathrm{CBF}$ between infants treated with FP and those given placebo, CBF data were combined to determine if there was an improvement with time (table 2). An insignificant trend for increased CBF was seen between weeks 0 and $12(\mathrm{p}=0.086)$.

\section{Transmission electron microscopy (TEM) findings}

A total of 77 specimens (32 placebo and $45 \mathrm{FP}$ ) were adequate for TEM. The proportions of collected specimens that could be examined by TEM at $0,3,6,12$, and 24 weeks were $7 / 12,7 / 13,6 / 13,7 / 13$, and $5 / 12$ in the placebo group and $12 / 16,12 / 15,11 / 14,6 / 11$, and $4 / 13$ in the FP group. As the results of TEM were similar in the two groups, the combined results are summarised in table 3. Assessment of cellular disruption as reflected by percentage of detached single cells showed virtually complete resolution by 6 weeks. A considerable percentage of dead epithelial cells was seen at baseline. The number of dead cells decreased over the next 3 weeks and had virtually disappeared by 6 weeks. TEM results of the control group have been reported previously ${ }^{5}$ and are summarised in table 3. These children had almost no detached single epithelial cells or dead cells in their nasal biopsy specimens, in stark contrast to those from infants with bronchiolitis.

A normal errors mixed model was use to investigate the decline in the percentage of ciliated cells showing abnormalities. The estimated time for the TEM epithelial ultrastructural parameters of each infant with bronchiolitis to return to the mean level of the control group was then computed and reported as the group estimated mean time in weeks for the percentage of ciliated cells with cilia losses (14.0 (range 10.0-20.8)), percentage of cells with cell projection abnormalities (16.7 (range 13.2-22.6)), percentage of cells with mitochondrial abnormalities (15.9 (range 11.619.1)), and epithelial integrity scores (13.1 (range 10.0$21.2)$ ). Figure 1 shows the decline in the epithelial integrity score. In no case was the rate of decline significantly affected by steroid treatment, although the tests have low power and there was a consistent but non-significant tendency for those on steroids to decline more quickly.

\section{DISCUSSION}

This is the first study to investigate the longitudinal changes in epithelial structure and ciliary activity of the nasal respiratory epithelium following acute bronchiolitis in infants. The first biopsy specimen was taken while the infants were recovering from their acute infection. This revealed severe epithelial damage, numerous dead cells, detached single cells, and almost total loss of cilia (fig 2). By 6 weeks the epithelial cells had returned to their columnar form but cellular projections were still present in half of

Table 3 Abnormal transmission electron microscopy findings

\begin{tabular}{|c|c|c|c|c|c|c|}
\hline \multirow[b]{2}{*}{ Abnormalities } & \multicolumn{6}{|l|}{ Weeks from baseline } \\
\hline & 0 & 3 & 6 & 12 & 24 & Normal * \\
\hline \multicolumn{7}{|l|}{ Cell types } \\
\hline Unciliated cells & $65.2(11.6), n=19$ & $53.7(19.1), n=19$ & $30.9(19.5), n=17$ & $23.7(10.5), n=13$ & $16.6(4.5), n=9$ & $21.0(9.5), n=16$ \\
\hline Mucous gland cells & $9.3(5.9), n=19$ & $9.1(4.4), n=19$ & $8.2(4.3), n=17$ & $10.7(4.0), n=13$ & $11.3(4.8), n=9$ & $9.9(4.8), n=16$ \\
\hline Dead cells & $9.1(5.4), n=19$ & $4.0(6.6), n=19$ & $0.4(1.8), n=17$ & $0.0(0.0), n=13$ & $0.0(0.0), n=9$ & $0.0(0.0), n=16$ \\
\hline \multicolumn{7}{|l|}{ Epithelial cells } \\
\hline \% ciliary lossest & $97.8(4.9), n=18$ & $86.0(13.6), n=17$ & $57.9(16.2), n=17$ & $30.5(17.0), n=13$ & $17.5(9.1), n=9$ & $24.1(10.1), n=16$ \\
\hline Projection & $85.4(7.0), n=19$ & $74.3(18.2), n=19$ & $46.4(16.3), n=17$ & $30.9(16.6), n=13$ & $16.0(6.4), n=9$ & $23.2(9.1), n=16$ \\
\hline Cytoplasmic blebs & $47.6(19.4), n=18$ & $31.4(17.3), n=19$ & $26.0(10.5), n=17$ & $18.8(9.2), n=13$ & $9.8(3.1), \mathrm{n}=9$ & $14.2(6.3), n=16$ \\
\hline Mitochondria & $59.7(12.6), n=19$ & $34.2(14.7), n=19$ & $21.6(8.8), n=17$ & $13.4(10.9), n=13$ & $10.6(4.1), n=9$ & $11.9(7.7), n=16$ \\
\hline Epithelial integrity & $3.8(0.9), n=19$ & $2.8(0.5), n=19$ & $1.7(0.5), n=17$ & $1.3(0.4), n=13$ & $0.7(0.4), n=9$ & $1.3(0.5), n=16$ \\
\hline \multicolumn{7}{|c|}{ Detached single cells (live+dead) } \\
\hline Ciliated cells & $9.3(6.4), n=19$ & $6.2(5.8), n=19$ & $2.3(5.3), n=17$ & $1.4(4.5), n=13$ & $0.0(0.0), n=9$ & $0.0(0.0), n=16$ \\
\hline Unciliated cells & $14.9(9.6), n=19$ & $6.2(9.9), n=19$ & $0.7(2.7), n=17$ & $0.4(1.3), n=13$ & $0.0(0.0), n=9$ & $0.0(0.0), n=16$ \\
\hline \multicolumn{7}{|l|}{ Ciliary ultrastructures } \\
\hline Dynein arms & $3.1(5.2), n=14$ & $1.2(2.1), \mathrm{n}=17$ & $0.9(1.2), \mathrm{n}=17$ & $1.5(1.5), n=13$ & $1.8(1.0), n=9$ & $1.8(3.2), \mathrm{n}=19$ \\
\hline Microtubule & $4.9(4.3), n=15$ & $3.5(3.7), n=17$ & $2.0(1.4), n=17$ & $1.7(1.6), n=13$ & $1.6(1.1), \mathrm{n}=9$ & $2.3(2.1), n=19$ \\
\hline
\end{tabular}

Values are shown as group mean (SD) percentage abnormality from infants with bronchiolitis over 24 weeks and from normal controls. *Normal children age $0-6$ years.

†Percentage ciliary losses in ciliated cells. 


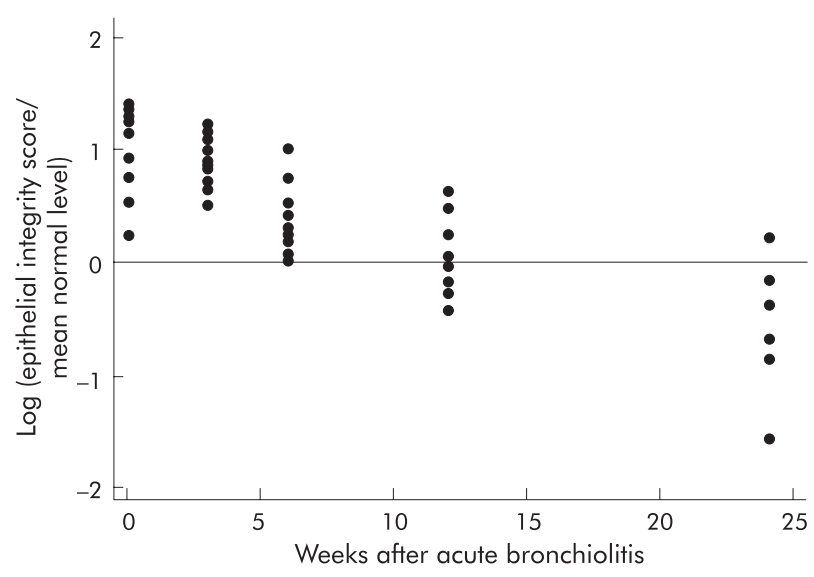

Figure 1 Decline in log epithelial integrity score over time. The line at zero represents the mean score in normal subjects which, on average, is reached by the treated infants after 13.1 weeks.

them. Although detached single cells and dead cells had largely disappeared, $58 \%$ of the ciliated epithelial cells continued to have evidence of ciliary loss. The number of ciliary ultrastructural abnormalities (dynein arm and microtubule damage) was small following bronchiolitis and diminished further with time. This is consistent with acute damage $^{1}$ as opposed to chronic damage where a more significant number of abnormalities would be expected. ${ }^{211}$ In children suffering from upper respiratory tract infections, microtubular abnormalities have been shown to be most evident during the first 2 weeks after the onset of illness. ${ }^{1}$ Following nasal inoculation of coronavirus into healthy adult volunteers, significant microtubular defects and ciliary dyskinesia were seen in biopsy specimens taken 3 days later. ${ }^{8}$ At the time of hospital discharge our infants would have been, on average, 3.5 days from admission and 8 days from the first sign of their illness. It is possible that more pronounced microtubular defects might have been found if cilia had been studied earlier in the illness. As it was impossible to determine the precise date of the onset of the illness, our statistical analysis took the biopsy time as the start time for estimating how long was needed for the ciliate epithelium to recover. However, it is unlikely that this would have increased our estimate of the recovery time by more that 8 days.

We have recently published data on the nasal epithelial ultrastructure from healthy children and infants with no known respiratory or ENT diseases. ${ }^{5}$ The baseline sample taken from the infants with bronchiolitis was the one that was most different from normal. Only the percentage of mucous cells remained normal throughout the follow up period. Using a normal errors mixed model, we were able to
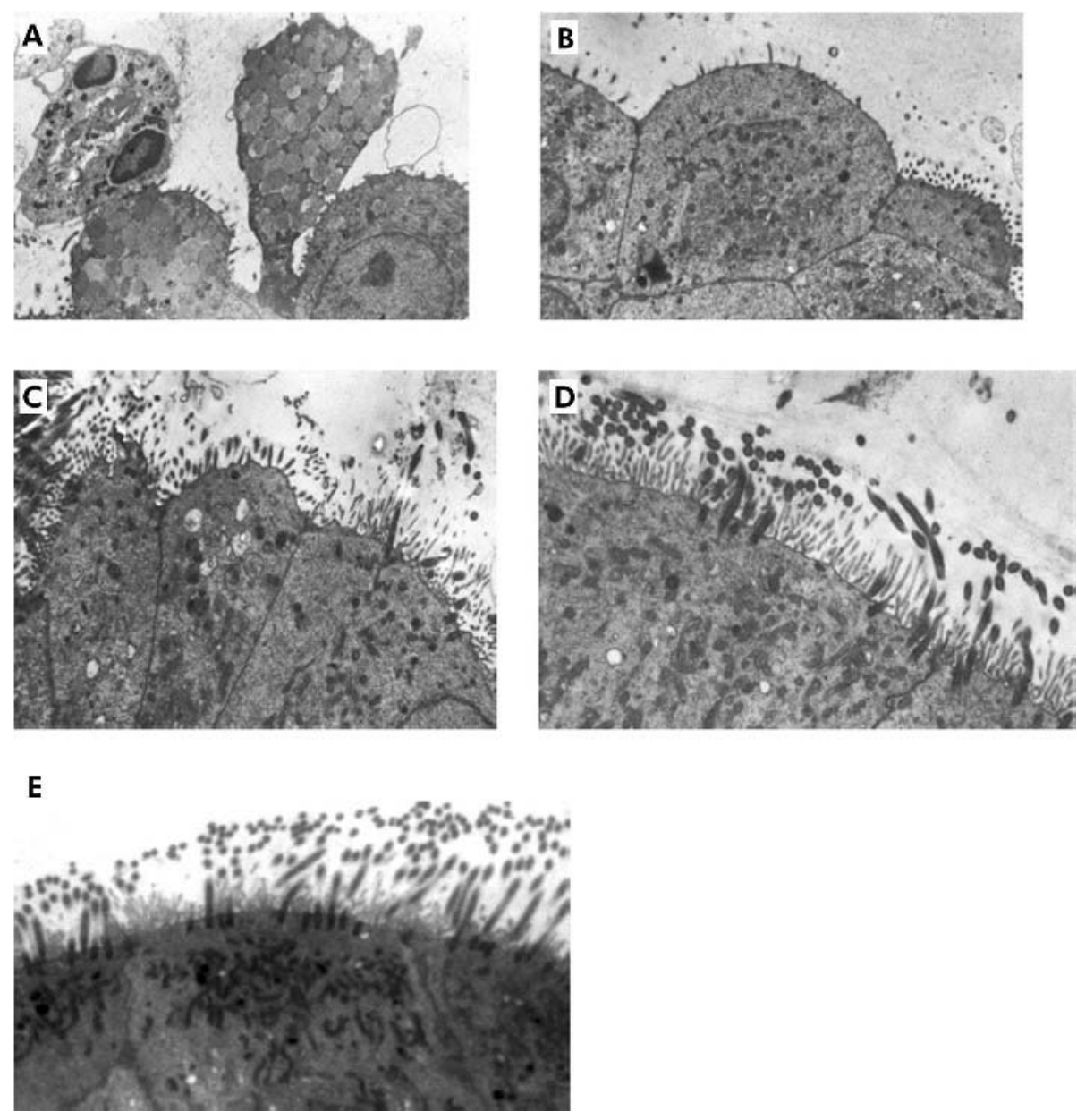

Figure 2 TEM changes of the nasal airway epithelium showing a gradual improvement in cilia and the respiratory epithelium over 6 months (24 weeks) in the same patient. (A) Week 0: gross disruption of the epithelium with projecting cells and mitochondrial damage; not cells are visible. (B) Week 3: Although no cilia are visible, the epithelium itself is intact; projection of cells is seen. (C) Week 6: a few cilia are seen and projection of cells is still apparent. (D) Week 12: projection of cells is no longer obvious in this specimen; increased numbers of cilia are seen. (E) Week 24: normal healthy epithelium with intact tight junction, no cellular projections and a normal complement of cilia. 
demonstrate normalisation of epithelial TEM parameters (percentage of ciliated cells showing cilia losses, cell projection abnormalities, mitochondrial abnormalities and epithelial integrity score except ciliary ultrastructure, mucous gland cells (which were obviously normal), and dead cells (not found in normal children)) 13-17 weeks after acute bronchiolitis compared with those expected in healthy 6 month old infants (an age similar to the infants with bronchiolitis towards the end of their 12 week period of inhaled treatment). Obviously, to determine the recovery time more accurately, further readings would be needed between 12 and 24 weeks. Our data did not demonstrate beneficial effects of inhaled corticosteroids on ciliary or epithelial recovery over the 12 week treatment period. Comparisons on percentage of dead cells were not performed as dead epithelial cells were not found on TEM of normal infants and children, which is obviously different from our data. Although traditional viral studies were performed, technical reasons may have accounted for the poor isolation rate. In addition, human metapneumovirus ${ }^{12}$ has only recently been described as a cause of bronchiolitis and it is possible that it was responsible for a proportion of our cases. Normal epithelial and ciliary TEM data in young children are scarce; in our reference normal group only five were infants. ${ }^{5}$ However, this is unlikely to be important as the percentage of TEM epithelial and ciliary ultrastructural abnormalities were essentially similar across all childhood age groups in our previous study.

Following bronchiolitis, many of the epithelial edges were found to lack or to have reduced numbers of cilia during the first 12 weeks. However, we were able to obtain at least four CBF readings per sample allowing some measure of the variation in readings obtained. Although CBF in our study was within normal limits, those measured during the first 36 weeks were slightly depressed. This is consistent with others who have reported only a slight reduction in CBF following rhinovirus, influenza $\mathrm{B}^{13}$ and coronavirus infections. ${ }^{8}$ As the ciliated epithelium became healthier over the study period, the confidence intervals of the CBF narrowed by $12-24$ weeks (table 2) towards those reported in our normal population (11.9 to 13.8$).{ }^{5}$ Ciliary ultrastructure in newborn ${ }^{14}$ and older children ${ }^{15}$ is similar to that in adults. We have, however, previously reported that CBF from infants in the immediate newborn period is slightly faster than in adults. ${ }^{15}$ This was not the case in children we have studied in infancy outside the neonatal period.

The photodiode technique is commonly used to measure CBF but does not give information on ciliary beat pattern. We have recently used a high speed video system to observe the ciliary beat pattern. ${ }^{16}{ }^{17}$ Following inoculation of healthy volunteers with coronavirus, we found a slight depression in CBF but the cilia observed were shown to beat in a dyskinetic fashion. Unfortunately, this system was not available at the time of our bronchiolitis study. It is possible that the extensive loss of cilia and the slow regeneration of the epithelial surface after bronchiolitis may play a more important role than the slightly diminished CBF in continuing post-bronchiolitis respiratory symptoms. ${ }^{4}$ Further studies are required to determine whether RSV infection causes ciliary dyskinesia and, if so, for how long after infection.

Our data suggest that bronchiolitic infections cause ciliary loss and may also open up intercellular tight junctions. This is reflected by the abnormal epithelial cell projection on TEM which lasted 16.7 weeks, although definitive proof of tight junction opening requires freeze fracture study of the epithelium. This suspicion is consistent with the hypothesis that RSV infections might open tight junctions, inviting bystander opportunistic aeroallergens to sensitise the airways and heighten bronchial responsiveness. ${ }^{18}$ Wilson and colleagues $^{13}$ showed a reduced nasal transmembrane potential difference in subjects infected with rhinovirus or influenza B virus, suggesting leaky epithelial cells following viral infection. Children with acute viral upper respiratory tract infections may have abnormal epithelial organisation and ciliary ultrastructure for up to 10 weeks. ${ }^{1}$ Acute bronchiolitis that is sufficiently severe to cause hospitalisation may represent a more severe spectrum of the disease or a disease process in vulnerable infants who may have residual abnormalities of the ciliary epithelium. It is also possible that a subsequent unrelated upper respiratory tract infection may have added effects in causing persistent/prolonged respiratory symptoms.

The aim of our clinical trial was to deliver inhaled steroids to the lower respiratory tract and we have previously shown that the device used was likely to deliver an adequate dose to the lungs. ${ }^{19}$ Although we were targeting the lung rather than the nose, it is likely that some of the inhaled steroids would also have deposited in the nose. However, the amount of inhaled steroids depositing in the nose of infants when breathing from the spacer device has not been studied. It is of interest that oral steroids used to treat asthmatic patients have been found to be beneficial in ciliogenesis. ${ }^{20}$ Our ciliary and epithelial data did not show obvious differences between the steroid treated and placebo groups.

In conclusion, denuded epithelial cells, abnormal epithelial cell projection, detached cells, and dead cells are seen in nasal epithelial biopsy specimens from infants following an episode of acute bronchiolitis requiring hospitalisation. Following acute bronchiolitis in infancy, the nasal epithelium takes 1317 weeks to return to normal.

\section{ACKNOWLEDGEMENTS}

The authors thank GlaxoWellcome for funding this project, the patients and their parents for taking part in the study, and Professor John Thompson, Leicester University and Dr Linda Hunt, University Department of Clinical Sciences, South Bristol for providing statistical assistance.

\section{Authors' affiliations}

J Y W Wong, A Rutman, C O'Callaghan, Department of Child Health, University of Leicester, School of Medicine, Leicester Royal Infirmary, Leicester LE2 7LX, UK

This study was funded by GlaxoWellcome.

\section{REFERENCES}

1 Carson JL, Collier AM, Hu SS. Acquired ciliary defects in nasal epithelium of children with acute viral upper respiratory infections. N Engl J Med 1985;312:463-8.

2 Giorgi PL, Oggiano N, Braga PC, et al. Cilia in children with recurrent upper respiratory tract infection: ultrastructural observations. Pediatr Pulmonol 1992;14:201-5.

3 Tristram DA, Hicks W Jr, Hard R. Respiratory syncytial virus and human bronchial epithelium. Arch Otolaryngol Head Neck Surg 1998;124:777-83.

4 Wong JY, Moon S, Beardsmore C, et al. No objective benefit from steroids inhaled via a spacer in infants recovering from bronchiolitis. Eur Respir J 2000;15:388-94

5 Chilvers MA, Rutman A, O'Callaghan C. Functional analysis of cilia and ciliated epithelial ultrastructure in healthy children and young adults. Thorax 2003:58:333-8.

6 Court SD. The definition of acute respiratory illness in children. Postgrad Med J 1973;49:771-6.

7 Yager J, Chen TM, Dulfano MJ. Measurement of frequency of ciliary beats of human respiratory epithelium. Chest 1978;73:627-33.

8 Chilvers MA, McKean M, Rutman A, et al. The effects of coronavirus on human nasal ciliated respiratory epithelium. Eur Respir J 2002;18:965-70.

9 Tsang KW, Rutman A, Tanaka E, et al. Interaction of Pseudomonas aeruginosa with human respiratory mucosa in vitro. Eur Respir $J$ 1994;7:1746-53.

10 Skrondal A, Rabe-Hesketh S. Generalized latent variable modeling: multilevel, longitudinal and structural equation models. Boca Raton, FL: Chapman \& Hall/CRC Press, 2003.

11 Rayner CF, Rutman A, Dewar A, et al. Ciliary disorientation in patients with chronic upper respiratory tract inflammation. Am J Respir Crit Care Med 1995; 151:800-4 
12 van den Hoogen BG, de Jong JC, Groen J, et al. A newly discovered human pneumovirus isolated from young children with respiratory tract disease. Nat Med 2001;7:719-24.

13 Wilson R, Alton E, Rutman A, et al. Upper respiratory tract viral infection and mucociliary clearance. Eur J Respir Dis 1987:70:272-9.

14 Barlow J, Wilkinson MJ, O'Callaghan C. Neonatal cilia: ultrastructure. Arch Dis Child 1990;65:708-10.

15 O'Callaghan C, Wilkinson MJ, Morgan D, et al. Neonatal cilial structure and function. Early Hum Dev 1990;22:109.

16 Chilvers MA, O'Callaghan C. Analysis of ciliary beat pattern and beat frequency using digital high speed imaging: comparison with the photomultiplier and photodiode methods. Thorax 2000;55:314-7.
17 Chilvers MA, Rutman A, O'Callaghan C. Ciliary beat pattern is associated with specific ultrastructural defects in primary ciliary dyskinesia. J Allergy Clin Immunol 2003;112:518-24.

18 Matsuse H, Behera AK, Kumar M, et al. Recurrent respiratory syncytial virus infections in allergen-sensitized mice lead to persistent airway inflammation and hyperresponsiveness. J Immunol 2000;164:6583-92

19 Wong J, Davies T, O'Callaghan C. Estimation of dose of fluticasone propionate inhaled by infants following bronchiolitis: effect on urinary cortisol excretion. J Allergy Clin Immunol 2002;110:721-7.

20 Heino M, Karjalainen J, Ylikoski J, et al. Bronchial ciliogenesis and oral steroid treatment in patients with asthma. Br J Dis Chest 1988;82 175-8.

\section{LUNG ALERT}

Mild persistent asthma: a pragmatic approach

$\Delta$ Boushey HA, Sorkness CA, King TS, et al. Daily versus as-needed corticosteroids for mild persistent asthma. N Engl J Med 2005;352:1519-28

T

his randomised, three arm, parallel, year long study compared the treatment of mild persistent asthma with budesonide $200 \mu \mathrm{g}$ bd, zafirlukast $20 \mathrm{mg}$ bd, or no controller

therapy. All patients were given a symptom based asthma treatment plan advising them to use open label budesonide or oral prednisone when their symptoms worsened. Patients had physician diagnosed asthma, and mild persistent disease was defined as self-treatment with $\beta$ agonists more than two days per week, night time awakenings related to asthma more than two days per month, or peak flow (PEF) variability of 20-30\%. Exclusion criteria included active smoking, respiratory tract infection or corticosteroid use in the previous 6 weeks, hospital admission or two or more emergency department visits for asthma in the previous year, or any features of moderate persistent asthma.

There was no significant difference in the primary outcome of change in morning PEF between the three groups. There were also no significant differences in the number of asthma exacerbations or in the asthma related quality of life scores. Patients treated with regular budesonide had 26 additional symptom-free days per year, a higher prebronchodilator $\mathrm{FEV}_{1}$, lower percentage of sputum eosinophils, lower exhaled nitric oxide levels, and higher asthma control scores than the two other groups. Although not powered as a non-inferiority study, these data suggest overtreatment of some patients with mild persistent asthma, with unnecessary exposure to the side effects of these drugs and additional healthcare expenditure. Providing patients with symptom based treatment plans individually tailored to the severity of their disease permits asthma control with a minimum of medication.

W Perera

Clinical Research Fellow, St Bartholomew's Hospital, London, UK; Wayomiperera@aol.com 